NETWORK WORKING GROUP

Request for Comments \#196

NIC 7141

Categories: A. 5, D.7

Obsoletes: none

Updates: none
Richard W. Watson

SRI-ARC

July 20, 1971

\title{
A MAIL BOX PROTOCOL
}

The purpose of this protocol is to provide at each site a standard mechanism to receive sequential files for immediate or deferred printing or other uses. The files for deferred printing would probably be stored on intermediate disk files, although details of how a file is handled, stored, manipulated, or printed at a site are not the concern of this protocol.

It is also assumed that there would be a program at the sending site which sends the file in the format given below with the optional control codes when appropriate. This program could probably be accessed as a subcommand of the Telnet program.

The motivation for developing this protocol is the Network Information Center's (NIC) need to be able to deliver messages and documents to remote sites, and to be able to receive documents for cataloging, redistribution, and other purposes from remote site without having to know the details of path name conventions and file system commands at each site. Multiple mail boxes (128) are allowed at each site and are identified as described below. The default is mail box number 0 for use with the standard mail printer defined below.

A mail box, as we see it, is simply a sequential file to which messages and documents are appended, separated by an appropriate site dependent code.

Although this protocol will enable people to transmit messages directly without going through the NIC, we want to encourage people to use the NIC as much as possible, so that dialogue will be recorded, cataloged and available for viewing online at NIC, using the powerful facilities of the ARC on Line system (NLS).

The Mail Box Protocol will use established network conventions, specifically the Network Control Program, Initial Connection Protocol, and Data Transfer Protocol, NIC 7104.

The normal transmission is to be full 7-bit ASCII in 8-bit bytes, the high order bit set to zero. 
The standard receiving mail printer for mail box number 0 is assumed to have a print line 72 characters wide, and a page of 66 lines. The new line convention will be carriage return ( $\mathrm{X}^{\prime} \mathrm{OD}^{\prime}$ ) followed by line feed $\left(\mathrm{X}^{\prime} \mathrm{OA}^{\prime}\right)$ as per the Telnet Protocol RFC 158, NIC 6768. The standard printer will accept form feed ( $\mathrm{X}^{\prime} \mathrm{OC}^{\prime}$ ) as meaning move paper to the top of a new page.

It is the senders responsibility to control the length of the print line and page. If more than 72 characters per line are sent or if more than 66 lines are sent without a form feed, than the receiving site can handle these situations as appropriate for them. These conventions can be changed by control codes as described below.

A message or document being sent to any mail box is a string of 8 bit bytes.

At the head of the message or document sent to mail box number 0 there is to be an initial address string terminated by a form feed. This address string is to contain the sender's name and address, and the receiver's name and address formatted in some reasonable, easy-to-read form for a clerk to read and distribute. Comments could also be included in the address string.

The format of information in mail boxes other than mail box number 0 is not explicitly defined by this protocol.

Initial Connection

Initial Connection will be as per the official Initial Connection Protocol, Documents \#2, NIC 7101, to a standard socket not yet assigned. A candidate socket number would be socket \#5.

Data Transmission

Data Transmission will be as per the Data Transfer Protocol, RFC 171, NIC 6793. That is, there will be a Modes Available handshake, and then transmission of special control

information and data. A message or document is defined to be a block of data. Control information is to be global. That is, once a control mode is set it is assumed to apply during the 
life of the connection unless explicitly changed. More than one document may be sent during the life of the connection unless the infinite bit stream mode is used. In the latter case there will be one message or document per connection. A reasonable convention for control information sent using the infinite bit stream mode seems to be to assume that is applies only to the next data stream connection from the host which sent the control stream.

Control Information

The sending process should be capable of allowing the user to indicate the control codes associated with the transmission of a mail item. The control codes can be used with any mail box number.

Mail Box Number

A site may find, as is the case at NIC, that it is useful to have more than one receiving mail box, each to be associated with a different process.

The mail box number for material to be printed by the standard mail printer is mail box number 0 and is used by default.

Code $\mathrm{X}^{\prime} \mathrm{DO} \mathrm{O}^{\prime}$

Meaning: A seven bit binary number in an eight bit field with the high order bit set to zero is to follow indicating the receiving mail box number.

Transmission Code Type

The default code type is 7-bit ASCII in an 8 bit field, high order bit to zero.

' Code $\mathrm{X}^{\prime} \mathrm{AO}$

Meaning: A Data Type signal indicating that the transmission code is 7-bit ASCII in an 8-bit field, high order set to zero. 
Code $\mathrm{X}^{\prime} \mathrm{A} 1^{\prime}$

Meaning: Transparency, i.e. a stream of 8 bit bytes.

Code $X^{\prime} A 2^{\prime}$

Meaning: EBCDIC

Other character codes could be added in the future.

Printer Control Codes

The default settings are a print line of 72 characters and a print page of 66 lines.

Code $X^{\prime} D 1$

Meaning: Set line width to 72 characters.

Code $\mathrm{X}^{\prime} \mathrm{D} 2^{\prime}$

Meaning: Use the full width of your printer.

Code $\mathrm{X}^{\prime} \mathrm{D} 3^{\prime}$

Meaning: Set page size to 66 lines.

Code $\mathrm{X}^{\prime} \mathrm{D} 4^{\prime}$

Meaning: Set page size to infinite.

Other virtual printer control codes can be added in the future.

Other classes of control codes can be added as the need arises.

[ This RFC was put into machine readable form for entry ]

[ into the online RFC archives by BBN Corp. under the ]

[ direction of Alex McKenzie. 12/96 ] 